\title{
Formation of Hierarchical Porous Structure via Breath Figure Method
}

\author{
Yongjian Zhang' and Zhiguang Li ${ }^{1}{ }^{2}$ \\ ${ }^{1}$ Shaanxi Key Laboratory of Surface Engineering and Remanufacturing, Xi'an University, Xian 710065, China \\ ${ }^{2}$ Key Laboratory of Eco-Textiles, Ministry of Education, College of Textiles and Clothing, Jiangnan University, Wuxi 214122, China \\ Correspondence should be addressed to Zhiguang Li; lizg@jiangnan.edu.cn
}

Received 6 December 2017; Accepted 7 February 2018; Published 20 March 2018

Academic Editor: Liyuan Zhang

Copyright ( 2018 Yongjian Zhang and Zhiguang Li. This is an open access article distributed under the Creative Commons Attribution License, which permits unrestricted use, distribution, and reproduction in any medium, provided the original work is properly cited.

\begin{abstract}
The porous structure films of PS containing silica nanoparticles of different hydrophobicity were obtained from breath figure method. For the porous films, the pores at the peripheral region and the center region were compared. It was found that the pores at the peripheral region appeared more uniform in size and showed higher degree of ordering. In addition, the patterns of the porous film became more disordered with the enhancing concentration of nanoparticles, and the pore size of the pore was increased. With the increase of the content of $\mathrm{SiOH}$, that is, decrease in particle hydrophobicity, the Si elements were aggregated at the surface of the film and the interior of the pores. A hierarchical porous structure had been obtained at appropriate humidity and particle concentration. Finally, a possible mechanism of the hierarchical structure was proposed.
\end{abstract}

\section{Introduction}

Breath figure $(\mathrm{BF})$ has attracted great attention to prepare patterned ordered porous polymer films because of its facile, economical, time saving, and convenient implementation [13]. The prepared porous films have large surface area and ordered porosity, which have been proved to be promising in a variety of applications in the fields of superhydrophobic surfaces, micropatterned templates, catalysis, resolution separation, and responsive surfaces [4-12].

The BF method is an easy process to fabricate honeycomb patterned porous films because the water droplets are applied as templates and easy removed by simple evaporation. In this process, polymers dissolved in a volatile and water-immiscible solvent are cast under high humidity. The rapid evaporation of volatile solvent can decrease the solution surface temperature, initiating the nucleation of water droplets onto the surface of the solution [13]. At the same time, the polymer aggregates at the interface between solution and water to stabilize the water droplets. Through thermocapillary effect and Marangoni convection, water droplets self-organize into close packed hexagonal arrays before their coagulation with each other [14, 15]. Eventually, after complete evaporation of the solvent and water droplets, hexagon-arranged honeycomb pores templating from water droplets are left on the polymer film surface. The surface morphologies of the porous films mainly depend on the polymer structure, humidity, concentration of solution, solvent, and the temperature of the solution surface [16-18]. A wide variety of polymers are devoted to obtain honeycomb structures. However, it is difficult to be applied if the polymer matrix requires complicated synthetic methods and high price. Polystyrene is a commercially available polymer, which is able to form porous structures by this process $[19,20]$.

Nanoparticles can be adsorbed at the fluid-fluid interfaces and significantly change the properties of these interfaces [2123]. Introducing nanoparticles into the polymer matrix leads to the preparation of new honeycomb patterned composite and excellent property. Sun et al. [24, 25] utilized polymer and silica particles to assist in preparing honeycomb porous films through BF method. Particles are proven to be effective in serving as stabilizers for making fine patterned porous arrays. However, the silica particles with varied surface hydrophobicity have not been investigated systematically yet. 
The wettability of ordered porous surfaces plays an important role in the practical applications. It is well known that the roughness of a surface can enhance the hydrophobic or hydrophilic properties. Ke et al. [26] prepared positively charged honeycomb films from PS-based block copolymer and silica particles by the breath figure method. The film surface is at a Cassie state where the negatively charged silica nanoparticles can selectively assemble on the external surface of films; however, silica nanoparticles assemble on both external and internal surfaces at a Wenzel state after prewetting. Consequently, by controlling the wetting states, nanoparticles selectively assemble inside or outside the pores influencing the wettability.

In addition to surface roughness, the investigations of surface chemistry of nanoparticles on the wettability are also important. In this paper, the porous structure films of the PS and silica nanoparticles of different surface hydrophobicity are fabricated from breath figure method. The surface morphology and the aggregation of $\mathrm{Si}$ elements of the porous structure films were investigated. The influences of important experimental factors, such as humidity and particle hydrophobicity, on the final morphology are discussed.

\section{Experimental}

2.1. Materials. Polystyrene (PS) $\left(M_{n}=1 \times 10^{5} \mathrm{~g} / \mathrm{mol}\right)$ was purchased from Aldrich. The silica particles used were fumed silica nanoparticles (Wacker Chemie), with primary diameter of $20-30 \mathrm{~nm}$. The hydrophobicity of the particles is characterized by the relative $\mathrm{SiOH}$ content on their surface. The hydrophobicity increases with a decrease of the content of $\mathrm{SiOH}$. In this study, the relative silanol content was $25 \%, 75 \%$, and $100 \%$, respectively. The solvent of chloroform $\left(\mathrm{CHCl}_{3}\right)$ was of analytical grade and used without further purification.

2.2. Preparation of Honeycomb Films. The honeycomb film was fabricated via BF technology $[18,27]$. The concentrations of $30 \mathrm{mg} / \mathrm{mL}$ of the $\mathrm{PS}$ and $\mathrm{SiO}_{2}$ particles $(1 \mathrm{mg} / \mathrm{mL}, 2 \mathrm{mg} / \mathrm{mL}$, and $5 \mathrm{mg} / \mathrm{mL})$ with different contents of $-\mathrm{OH}(25 \%, 75 \%$, and $100 \%)$ were dissolved in chloroform solution and then cast onto a silicon wafer under a humid airflow. After the complete evaporation of solvent and water droplets, porous film was obtained.

2.3. Characterization. Surface morphologies of the films were characterized by optical microscopy (XJX-2, Nanjing, China) and a scanning electron microscope (SEM) carried on VEGA $3 \mathrm{LMH}$ (Česko TESCAN) with $20 \mathrm{kV}$ accelerating voltage. The quantitative analysis was using energy dispersive spectrometry (EDS) (Oxford INCA X-ACT). The water contact angle was tested using the sessile drop method (JC2000D4 Powereach Tensiometer).

\section{Results and Discussion}

3.1. Spatial Variation of Porous Structures. Before the BF process, the PS solution drop was deposited on the glass slide. The morphology of the drop is shown in Figure 1(a). The contact angle of the drop was measured to be $\sim 15.5^{\circ}$. The peripheral area was marked as region $\mathrm{B}$ and the center area was region $C$. The SEM images of region $B$ and region $C$ are shown in Figures 1(b) and 1(c), respectively.

For the hole structure of the porous films, the morphology at the peripheral area is noticeably different from that at the center area. At the peripheral area, honeycomb like ordered pores with uniform sizes are observed. In contrast, the pores formed at the center of the films are evidently less ordered and the size distribution is much broader than that at the peripheral area. The hole is distributed in a monolayer at the periphery of the drop, whereas at the center part, multilayers of holes are examined. Moreover, the size of holes becomes multidispersed.

The differences in the pore morphology between the peripheral and center regions resulted from the difference in evaporation rate at different regions.

During the fast evaporation of the $\mathrm{CHCl}_{3}$, Marangoni convection can actively transport most of the PS together with the water droplets from center to edge of the solution drop. Thus, the effective concentration of the PS is higher near the contact lines than that at the center of the solution drop [28]. During the volatilization of solvent, the contact line of the solution is pinned, possibly due to both surface roughness of the substrate and the deposition of the PS [29]. The pinning effect depends on the initial contact angle of the deposited solution on the silicon wafer substrate [30]. The evaporation rate is higher at the periphery than at the center. Correspondingly, the faster vitrification at the peripheral region also helps to limit the growth and coalescence of the trapped water droplets [31]. In comparison, the delayed gelation/vitrification at the center region results in longer growth and coalescence time for the water droplets, and thus the larger and polydisperse pores.

3.2. Influence of Nanoparticles. With the presence of particles, a particle layer could form at the water solution interface which possesses shear and dilational moduli [21]. The mechanical properties of the particle layer can influence the growth of droplets. It is reasonable to expect smaller droplet size corresponding to stronger particle layer, which is indeed in line with the previous rheological study of the particle layer [32]. Figure 2 shows different patterns using different concentrations of the silica nanoparticles with the same PS solution.

It is obvious that the patterns of the film surface become increasingly disordered with greater nanoparticle concentration. The ordered hexagonal arrangement of the pores has been compromised with increasing particle concentration, and the pore size is larger for higher application quantity of the particle. When the particle concentration exceeds a critical value above one concentration, the porous films are not formed. This is because the adsorption of too much nanoparticles at the water droplet surface may result in a jamming state to the interface, which hinders the droplet growth and leads to the formation of irregular shaped droplets instead of uniform droplet pattern [33].

At an appropriate particle concentration, ordered porous structure can be obtained. However, the average pore size depends on the hydrophobicity of the particles. The SEM 


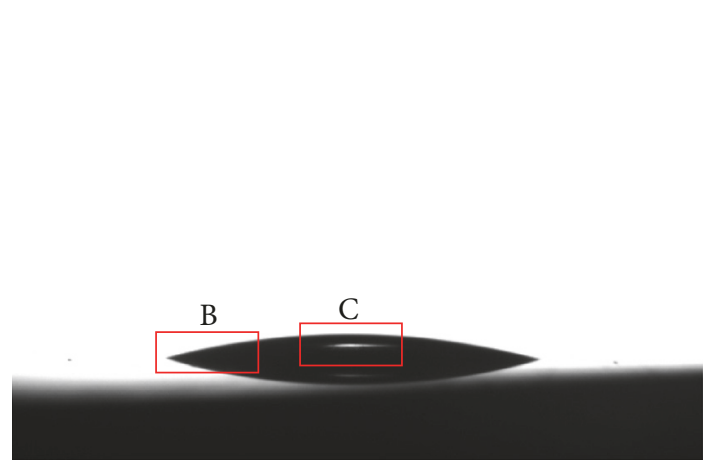

(a)

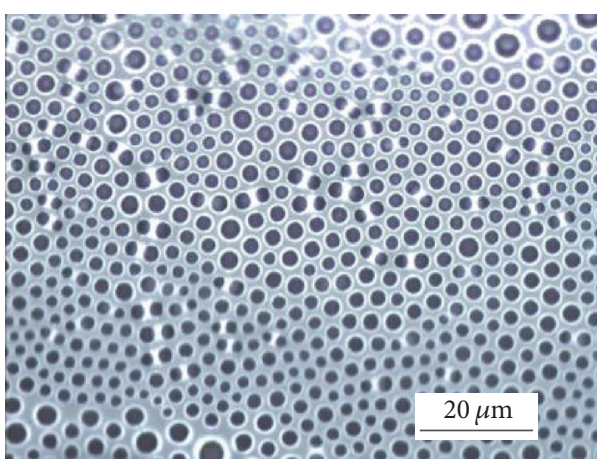

(b)

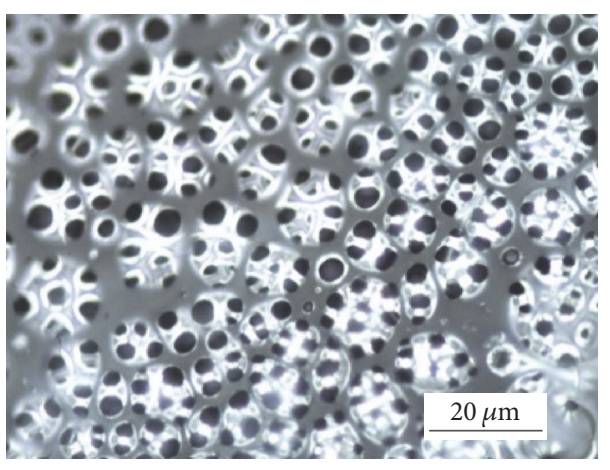

(c)

FIGURE 1: Spatial dependence of the porous structure obtained by BF method using PS solution ( $30 \mathrm{mg} / \mathrm{mL})$. (a) Initial morphology of the PS solution drop; ((b) and (c)) the formed porous structures at the peripheral and center of the drop through breath figure method, respectively, as marked in (a).

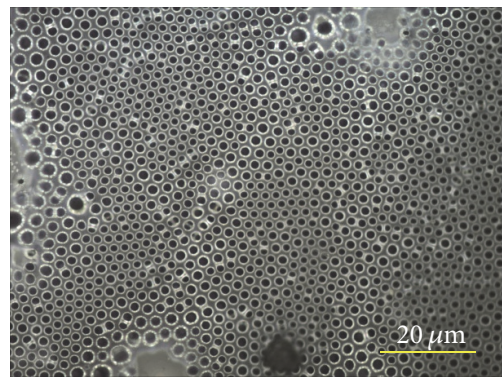

(a)

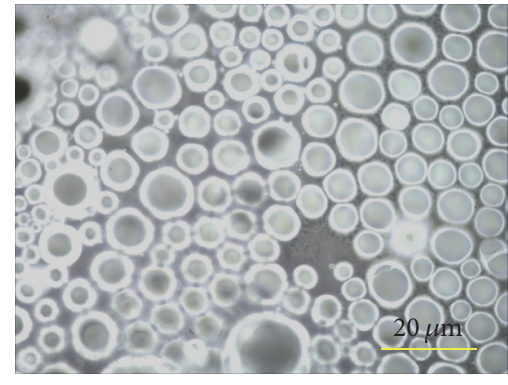

(b)

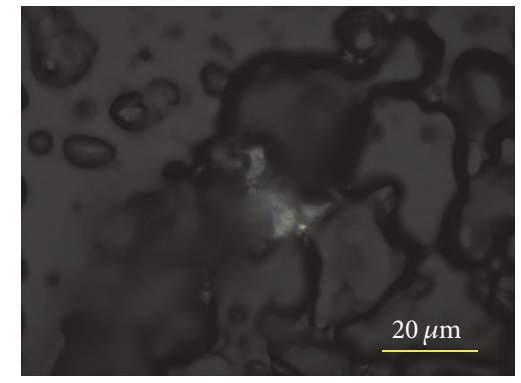

(c)

FIGURE 2: Influence of the nanoparticle ( $37 \% \mathrm{SiOH})$ concentration on the final porous structures obtained from the PS solution (30 $\mathrm{mg} / \mathrm{mL})$. (a) $1 \mathrm{mg} / \mathrm{mL}$, (b) $2 \mathrm{mg} / \mathrm{mL}$, and (c) $5 \mathrm{mg} / \mathrm{mL}$.

images of the porous structure with particles of varied hydrophobicity are shown in Figure 3. The average pore size as a function of the hydrophobicity particles of the porous films are shown in Figure 4. It is noteworthy that the average pore sizes of the obtained films decrease from $0.83 \mu \mathrm{m}, 1.09 \mu \mathrm{m}$ to $1.29 \mu \mathrm{m}$ when the $\mathrm{SiOH}$ contents increase from $25 \%$ to $100 \%$. With the increase of $-\mathrm{OH}$ contents, the pore sizes are increased. It indicates that when the PS is fixed, the augment of units of -OH will enhance the capability of holding water during the water-organic interface, which enlarges the pore size. On the other hand, the existence of hydrophilic segment is beneficial to stabilize the water droplets at the interface, resulting in forming more ordered porous film. Hydrophilic segment is known to form inverse structures in organic solvent [34]. When contacting with water, these inverse structures start interacting with water, leading to a rearrangement of PS around the water droplets. With the evaporation of the solvent and water, the pores enriched with hydrophilic functionality are formed.

In order to investigate where the particles were distributed, EDS was utilized and the Si elements were marked as red dots in Figure 3. For the most hydrophobic particle used in the present work $(25 \% \mathrm{SiOH})$, most of the particle are distributed at the rim of the pores. With the increase of 


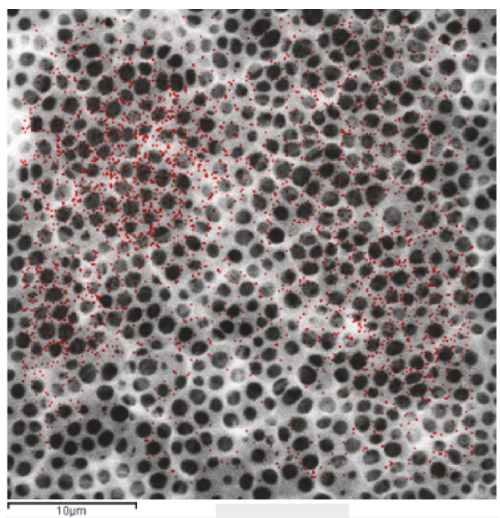

(a)

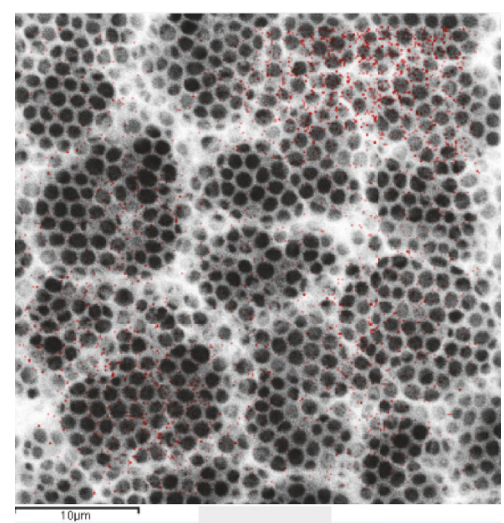

(b)

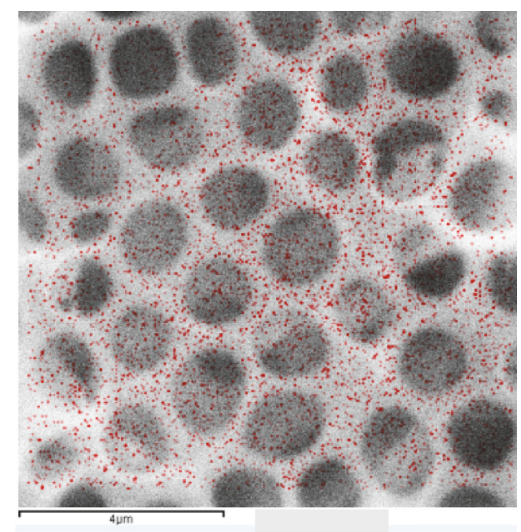

(c)

Figure 3: Influence of particle hydrophobicity concentration on the final porous structures. (a) $25 \% \mathrm{SiOH}$, (b) $75 \% \mathrm{SiOH}$, and (c) $100 \% \mathrm{SiOH}$. The red dots are the distribution of Si element.

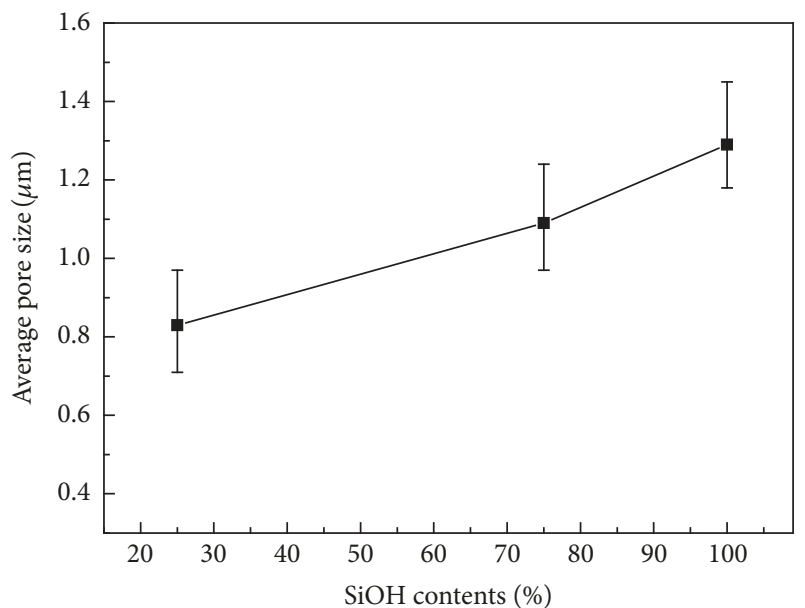

FIGURE 4: The average pore size as a function of the $\mathrm{SiOH}$ contents.

$\mathrm{SiOH}(100 \% \mathrm{SiOH})$, the particles also appear at the surface of the film and the interior of the pores. This is mainly attributed to the hydrophilic nature of these particles.

3.3. Formation of Hierarchical Porous Structure. When humidity is increased to $95 \%$, the hierarchical porous structure has been obtained in Figure 5, which is characterized by large porous pores containing many small holes in their interiors with $D_{\mathrm{S}} / D_{\mathrm{L}} \approx 1 / 10\left(D_{\mathrm{S}}\right.$ and $D_{\mathrm{L}}$ are the diameter for small and large holes, resp.).

This hierarchical porous structure can only be formed at high relative humidity $(\mathrm{RH}=95 \%)$. In this case, coalescence among water droplets could be enhanced, consequently leading to polydispersity in droplet sizes [35]. The formation mechanism can be illustrated in Figure 6. The water droplets approach on the surface of the polymer solution (Figure 6(a)). The first unstable layer of water droplets was condensed at the solution surface as shown in Figure 6(b). Due to the high $\mathrm{RH}$, the droplets close to the first layer condensation have coalesced to larger droplets. With the volatilization of

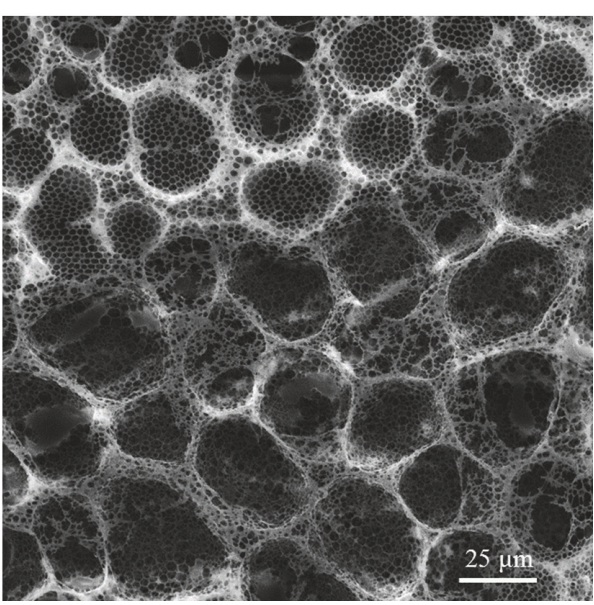

FIgURE 5: Hierarchical porous structure obtained at $\mathrm{RH}=95 \%$.

solvent and water droplets, the smaller pores with metastable state are formed in Figure 6(c). The coalesced larger water droplets are dragged into the polymer solution through a thermocapillary effect, and then the large pores are formed as shown in Figure 6(d). The first unstable layer is squeezed to form a spherical shape because of the gravity of the larger water droplet. This process can repeat to form multilayer structure until the solvent and water droplet are thoroughly evaporated (Figure 6(e)).

The size of the periodic microstructures in honeycomb porous falls within the range of hundreds of nanometers to tens of micrometers, matching the requirements of templates, separation, cell culture, biosensing, optical and optoelectronic devices, and so on $[1,36-38]$.

\section{Conclusions}

In this paper, the porous films of PS and silica nanoparticles of different $-\mathrm{OH}$ are prepared through breath figure method. For all the porous films obtained, the pore morphology at 


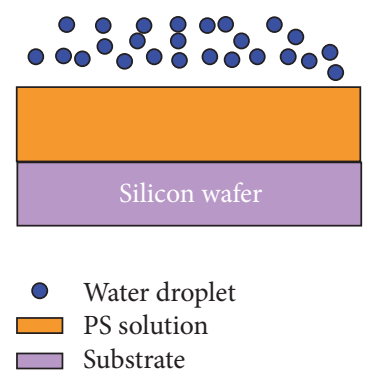

(a)

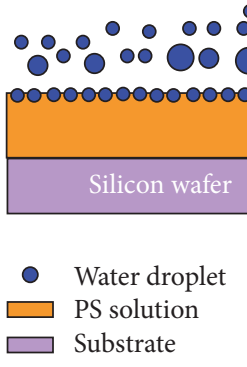

(b)

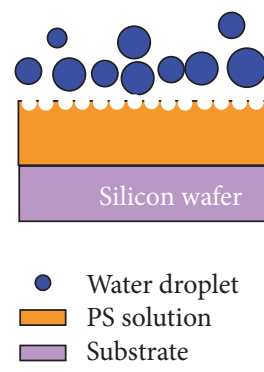

(c)

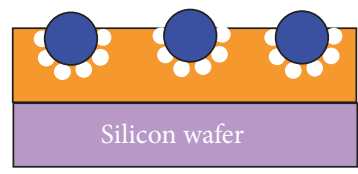

- Water droplet PS solution Substrate

(d)

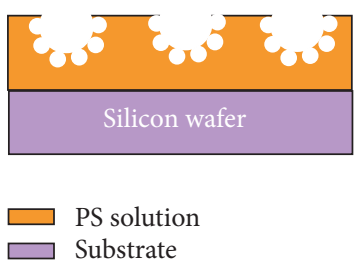

(e)

FIGURE 6: Schematic illustration for the formation of the hierarchical structure. (a) Water droplets approaching the PS solution. (b) Condensation of the first layer droplets. (c) Coalescence of the larger droplets. (d) Successive condensation and sedimentation of the coalesced droplets. (e) Formation of the final hierarchical structure.

the peripheral regions is found more uniform and ordered than that at the center region. Such a difference is attributed to the interplay between the contact line pinning and the evaporation rate. The patterns of the film become more disordered with the enhancing concentration of nanoparticles, and the pore size is increased. Moreover, the average pore size and the distribution of particles depend on the particle hydrophobicity. With the increase of $\mathrm{SiOH}$ content, the pore size tends to increase and the silica nanoparticle seems aggregate at the surface of the film and the interior of the pores. Interestingly, when the humidity is increasing to $95 \%$, the hierarchical porous structure was observed, which is characterized by larger porous pores containing many small holes in the interior. This hierarchical porous structure is probably due to the humidity enhanced droplet coalescence and the thermocapillary effect caused by evaporation.

\section{Conflicts of Interest}

The authors declare that there are no conflicts of interest regarding the publication of this paper.

\section{Acknowledgments}

This work was supported by the Foundation of Shaanxi Provincial Education Department, China (Grant no. 17JK1123), the Xi'an Science and Technology Project (Grant no. 2017CGWL15), and the Fundamental Research Funds for the Central Universities (Grant no. JUSRP11702). The authors thank Mr. H. B. Li and Mr. X. Lu for the help in experiments.

\section{References}

[1] A. J. Zhang, H. Bai, and L. Li, "Breath figure: a natureinspired preparation method for ordered porous films," Chemical Reviews, vol. 115, no. 18, pp. 9801-9868, 2015.

[2] L.-S. Wan, L.-W. Zhu, Y. Ou, and Z.-K. Xu, "Multiple interfaces in self-assembled breath figures," Chemical Communications, vol. 50, no. 31, pp. 4024-4039, 2014.

[3] A. Muñoz-Bonilla, M. Fernández-García, and J. RodríguezHernández, "Towards hierarchically ordered functional porous polymeric surfaces prepared by the breath figures approach," Progress in Polymer Science, vol. 39, no. 3, pp. 510-554, 2014.

[4] Z. Li, X. Ma, Q. Kong, D. Zang, X. Guan, and X. Ren, "Static and Dynamic Hydrophobic Properties of Honeycomb Structured Films via Breath Figure Method," The Journal of Physical Chemistry C, vol. 120, no. 33, pp. 18659-18664, 2016.

[5] Q. Hong, X. Ma, Z. Li, F. Chen, and Q. Zhang, "Tuning the surface hydrophobicity of honeycomb porous films fabricated by star-shaped POSS-fluorinated acrylates polymer via breathfigure-templated self-assembly," Materials \& Design, vol. 96, pp. $1-9,2016$.

[6] P. Zhang, H. Chen, L. Zhang, T. Ran, and D. Zhang, “Transparent self-cleaning lubricant-infused surfaces made with largearea breath figure patterns," Applied Surface Science, vol. 355, pp. 1083-1090, 2015

[7] L.-S. Wan, Q.-L. Li, P.-C. Chen, and Z.-K. Xu, "Patterned biocatalytic films via one-step self-assembly," Chemical Communications, vol. 48, no. 37, pp. 4417-4419, 2012.

[8] H. Yuan, B. Yu, H. Cong et al., "Preparation of highly permeable BPPO microfiltration membrane with binary porous structures on a colloidal crystal substrate by the breath figure method," Journal of Colloid and Interface Science, vol. 461, pp. 232-238, 2016. 
[9] J. Mansouri, E. Yapit, and V. Chen, "Polysulfone filtration membranes with isoporous structures prepared by a combination of dip-coating and breath figure approach," Journal of Membrane Science, vol. 444, pp. 237-251, 2013.

[10] C. Du, A. Zhang, H. Bai, and L. Li, "Robust microsieves with excellent solvent resistance: Cross-linkage of perforated polymer films with honeycomb structure," ACS Macro Letters, vol. 2, no. 1, pp. 27-30, 2013.

[11] A. S. De León, M. Molina, S. Wedepohl, A. Muñoz-Bonilla, J. Rodríguez-Hernández, and M. Calderón, "Immobilization of Stimuli-Responsive Nanogels onto Honeycomb Porous Surfaces and Controlled Release of Proteins," Langmuir, vol. 32, no. 7, pp. 1854-1862, 2016.

[12] P. Escalé, W. Van Camp, F. Du Prez, L. Rubatat, L. Billon, and M. Save, "Highly structured $\mathrm{pH}$-responsive honeycomb films by a combination of a breath figure process and in situ thermolysis of a polystyrene-block-poly(ethoxy ethyl acrylate) precursor," Polymer Chemistry, vol. 4, no. 17, pp. 4710-4717, 2013.

[13] Y.-C. Chiu, C.-C. Kuo, C.-J. Lin, and W.-C. Chen, "Highly ordered luminescent microporous films prepared from crystalline conjugated rod-coil diblock copolymers of PF-b-PSA and their superhydrophobic characteristics," Soft Matter, vol. 7, no. 19, pp. 9350-9358, 2011.

[14] N. Maruyama, T. Koito, J. Nishida et al., "Mesoscopic patterns of molecular aggregates on solid substrates," Thin Solid Films, vol. 327-329, no. 1-2, pp. 854-856, 1998.

[15] M. Srinivasarao, D. Collings, A. Philips, and S. Patel, "Threedimensionally ordered array of air bubbles in a polymer film," Science, vol. 292, no. 5514, pp. 79-83, 2001.

[16] H. Wu, T. Jiang, J. Zhu, K. Cui, Q. Zhao, and Z. Ma, "Synthesis of poly(ethylene-co-vinyl alcohol)-g-polystyrene graft copolymer and their applications for ordered porous film and compatibilizer," Journal of Polymer Science Part A: Polymer Chemistry, vol. 54, no. 4, pp. 516-524, 2016.

[17] L.-W. Zhu, L.-S. Wan, J. Jin, and Z.-K. Xu, "Honeycomb porous films prepared from porphyrin-cored star polymers: Submicrometer pores induced by transition of monolayer into multilayer structures," The Journal of Physical Chemistry C, vol. 117, no. 12, pp. 6185-6194, 2013.

[18] Z. Li, Z. Zhang, Q. Kong, and X. Ren, "Adhesive and repulsive properties of water droplet impact on honeycomb surfaces through breath figure method," Journal of Applied Polymer Science, vol. 134, no. 47, Article ID 45476, 2017.

[19] L.-W. Zhu, W. Yang, Y. Ou, L.-S. Wan, and Z.-K. Xu, "Synthesis of polystyrene with cyclic, ionized and neutralized end groups and the self-assemblies templated by breath figures," Polymer Chemistry, vol. 5, no. 11, pp. 3666-3672, 2014.

[20] E. Ferrari, P. Fabbri, and F. Pilati, "Solvent and substrate contributions to the formation of breath figure patterns in polystyrene films," Langmuir, vol. 27, no. 5, pp. 1874-1881, 2011.

[21] D. Y. Zang, E. Rio, D. Langevin, B. Wei, and B. P. Binks, "Viscoelastic properties of silica nanoparticle monolayers at the air-water interface," European Physical Journal E, vol. 31, no. 2, pp. 125-134, 2010.

[22] Z. Chen, D. Zang, L. Zhao et al., "Liquid Marble Coalescence and Triggered Microreaction Driven by Acoustic Levitation," Langmuir, vol. 33, no. 25, pp. 6232-6239, 2017.

[23] D. Zang, Y. Yu, Z. Chen, X. Li, H. Wu, and X. Geng, "Acoustic levitation of liquid drops: Dynamics, manipulation and phase transitions," Advances in Colloid and Interface Science, vol. 243, pp. 77-85, 2017.
[24] W. Sun, Y. Zhou, Y. Ju, L. Yang, T. Xu, and Z. Chen, "A study of morphology modulation of honeycomb hybrid films and the interfacial behavior of silica particles within a patterned polymeric matrix," Macromolecular Chemistry and Physics, vol. 215, no. 1, pp. 96-102, 2014.

[25] W. Sun, Z. Shao, and J. Ji, "Particle-assisted fabrication of honeycomb-structured hybrid films via breath figures method," Polymer, vol. 51, no. 18, pp. 4169-4175, 2010.

[26] B.-B. Ke, L.-S. Wan, P.-C. Chen, L.-Y. Zhang, and Z.-K. Xu, "Tunable assembly of nanoparticles on patterned porous film," Langmuir, vol. 26, no. 20, pp. 15982-15988, 2010.

[27] Z. Li, Q. Kong, X. Ma, D. Zang, X. Guan, and X. Ren, "Dynamic effects and adhesion of water droplet impact on hydrophobic surfaces: Bouncing or sticking," Nanoscale, vol. 9, no. 24, pp. 8249-8255, 2017.

[28] L. Wang, S. H. Maruf, D. Maniglio, and Y. Ding, "Fabrication and characterizations of crosslinked porous polymer films with varying chemical compositions," Polymer, vol. 53, no. 17, pp. 3749-3755, 2012.

[29] R. D. Deegan, O. Bakajin, T. F. Dupont, G. Huber, S. R. Nagel, and T. A. Witten, "Contact line deposits in an evaporating drop," Physical Review E, vol. 62, no. 1 B, pp. 756-765, 2000.

[30] T. Kajiya, C. Monteux, T. Narita, F. Lequeux, and M. Doi, "Contact-line recession leaving a macroscopic polymer film in the drying droplets of water-poly(N, N-dimethylacrylamide) (PDMA) solution," Langmuir, vol. 25, no. 12, pp. 6934-6939, 2009.

[31] T. Nishikawa, R. Ookura, J. Nishida et al., "Fabrication of honeycomb film of an amphiphilic copolymer at the air-water interface," Langmuir, vol. 18, no. 15, pp. 5734-5740, 2002.

[32] D. Y. Zang, E. Rio, G. Delon, D. Langevin, B. Wei, and B. P. Binks, "Influence of the contact angle of silica nanoparticles at the air-water interface on the mechanical properties of the layers composed of these particles," Molecular Physics, vol. 109, no. 7-10, pp. 1057-1066, 2011.

[33] W. Sun, Y. Zhou, and Z. Chen, "Fabrication of honeycombstructured porous film from polystyrene via polymeric particleassisted breath figures method," Macromolecular Research, vol. 21, no. 4, pp. 414-418, 2013.

[34] X.-Y. Li, Q.-L. Zhao, T.-T. Xu, J. Huang, L.-H. Wei, and Z. Ma, "Highly ordered microporous polystyrene-b-poly(acrylic acid) films: Study on the influencing factors in their fabrication via a static breath-figure method," European Polymer Journal, vol. 50, no. 1, pp. 135-141, 2014.

[35] C. Wang, Y. Mao, D. Wang, Q. Qu, G. Yang, and X. Hu, "Fabrication of highly ordered microporous thin films by PSb-PAA self-assembly and investigation of their tunable surface properties," Journal of Materials Chemistry, vol. 18, no. 6, pp. 683-690, 2008.

[36] B. Yao, Q. Zhu, L. Yao, and J. Hao, "Fabrication of honeycombstructured poly(ethylene glycol)-block-poly(lactic acid) porous films and biomedical applications for cell growth," Applied Surface Science, vol. 332, pp. 287-294, 2015.

[37] G. Leone, U. Giovanella, F. Bertini et al., "Hierarchically structured, blue-emitting polymer hybrids through surfaceinitiated nitroxide-mediated polymerization and water templated assembly," Journal of Materials Chemistry C, vol. 1, no. 40, pp. 6585-6593, 2013.

[38] C. Zhang, X. Wang, K. Min et al., "Developing porous honeycomb films using miktoarm star copolymers and exploring their application in particle separation," Macromolecular Rapid Communications, vol. 35, no. 2, pp. 221-227, 2014. 

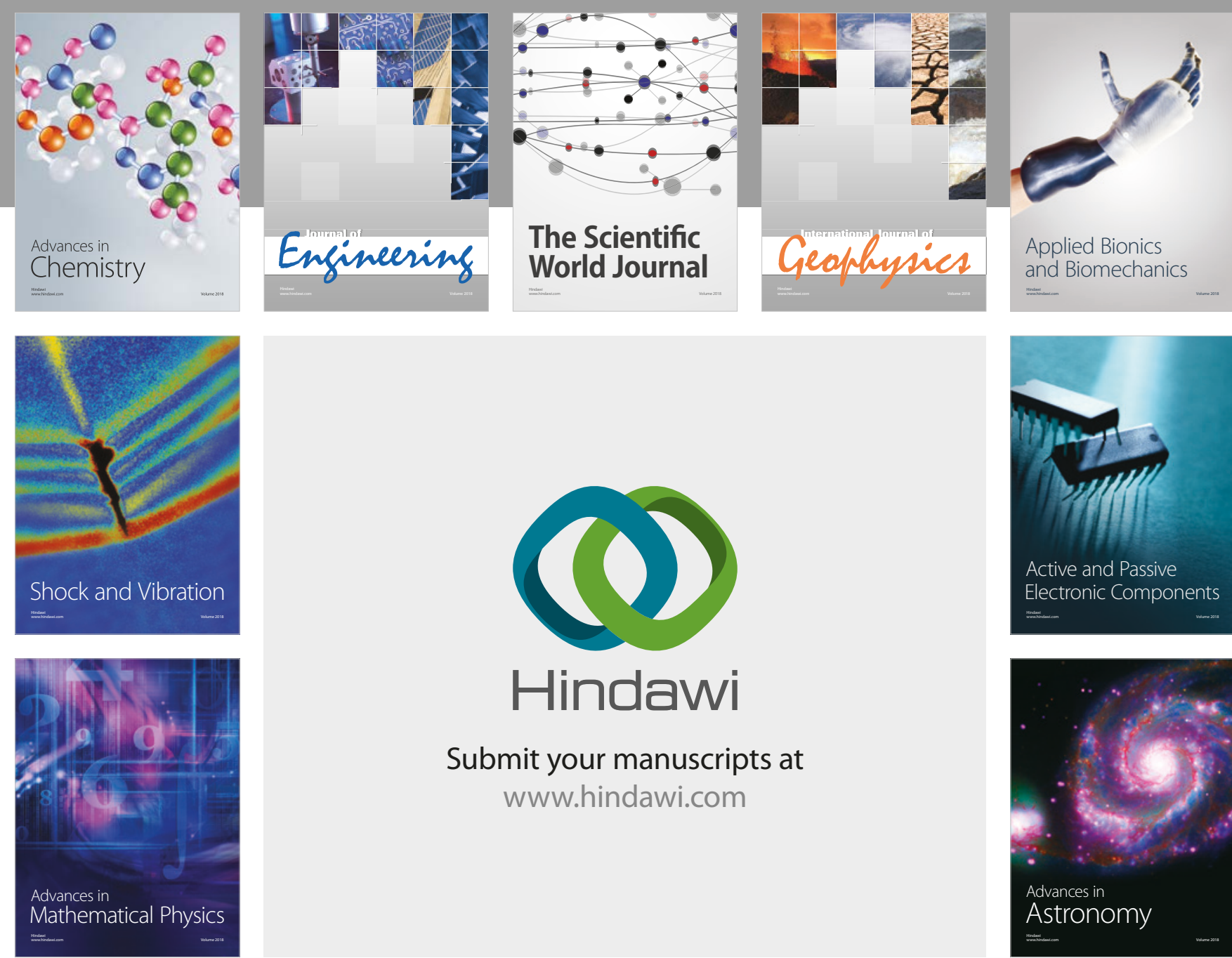

Submit your manuscripts at

www.hindawi.com

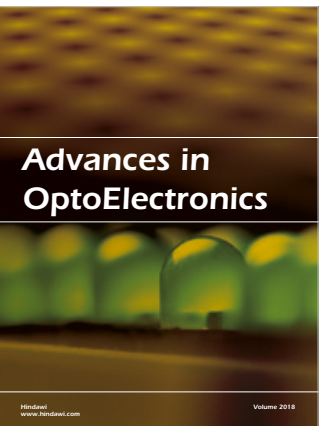

\section{Rotcting Machinery}
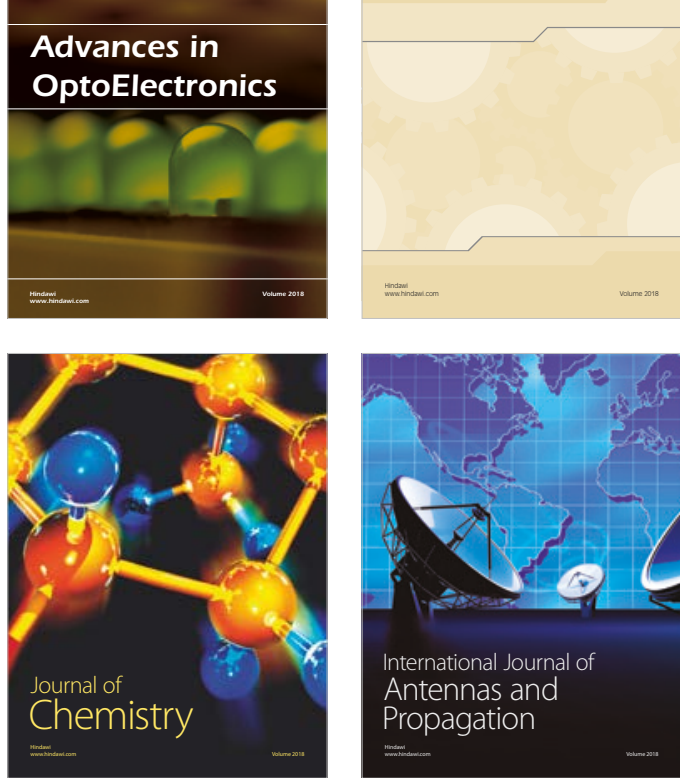

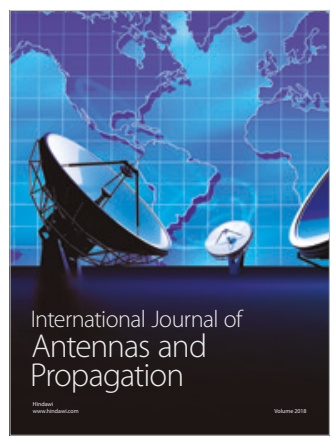

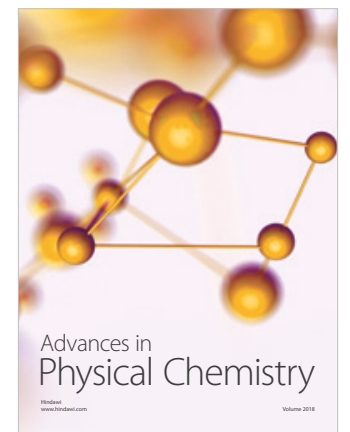

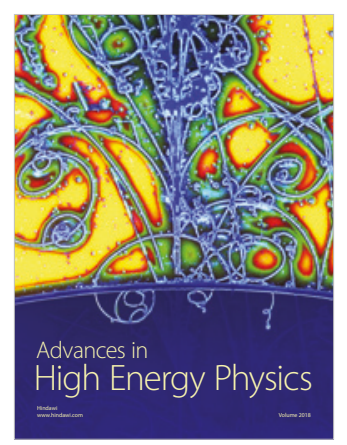

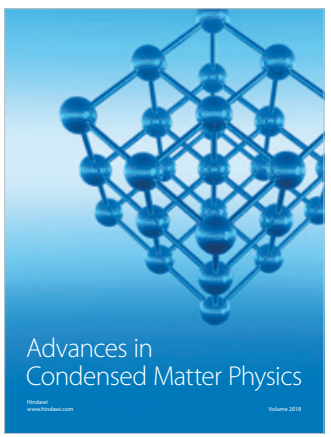

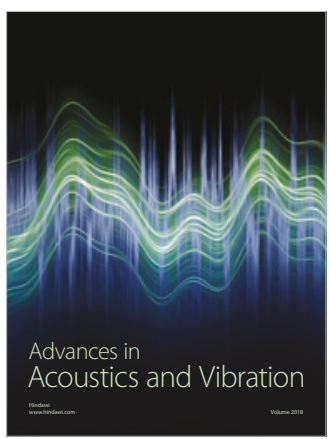

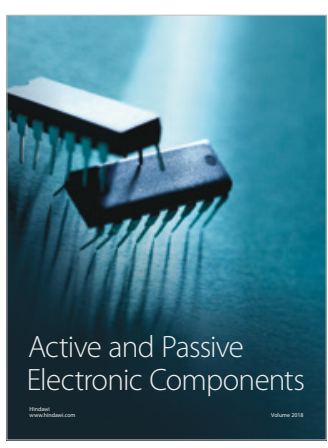
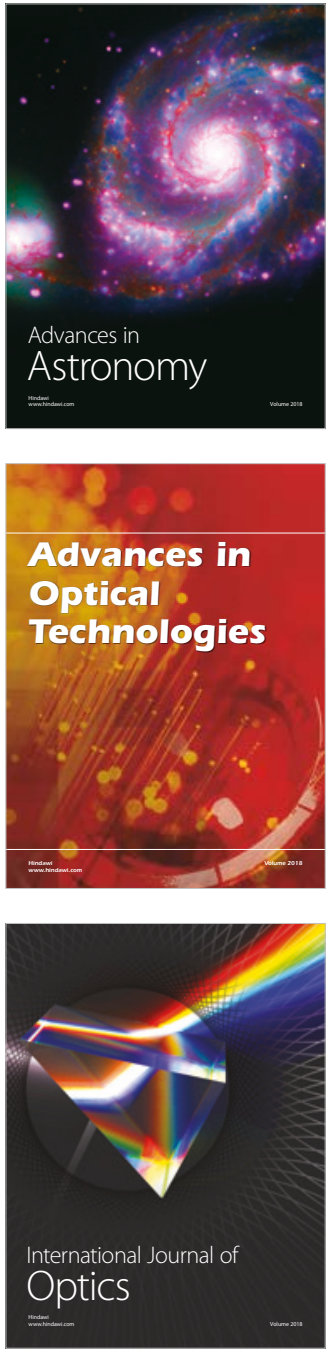